\title{
Correlation of Hand Ischemic Questionnaire with Digital Brachial Index In Patient with Upper Arm Native Arteriovenous Fistula \\ Raden Suhartono ${ }^{*}$, Muhammad Relly Sofiar ${ }^{b}$, Aria Kekalih ${ }^{c}$
}

Introduction: Steal syndrome is one of the most feared complications in maintaining arteriovenous fistula (AVF) for hemodialysis. The incidence of steal syndrome in worldwide is estimated to be $0.5-5 \%$. There are various non-invasive examinations to assess the degree of stealing, one of which is the digital brachial index (DBI). In this study, subjects with brachiocephalic AVF were assessed by the hand ischemic questionnaire (HIQ) to assess the manifestations of steal syndrome complained by the patient, which are the sensation of cold, pain, the decrease in sensation and strength, and cramps. The literature about the correlation of DBI with other assessment is still limited.

Method: The subjects of this study were all patients undergoing hemodialysis with upper arm AVF at Cipto Mangunkusumo Hospital in the period May - June 2019. Patients will be asked about various symptoms of stealing syndrome, the severity, and also frequency, according to $\mathrm{HIQ}$. The scores were then calculated, followed by scores of DBI measurements using a plethysmograph. The DBI values that considered to be meaningful as stealing syndrome were $<0.6$. The correlation between the two parameters was then analyzed.

Results: From demographic data, characteristics of patients with native AVF by sex were 37 (46.2\%) men and 43 (53.8\%) women with an average age of 53 years. The minimum value of the HIQ score was 0 , and the maximum value was 70 , with a median value of 3 . The correlation test between the total value of the HIQ questionnaire score and the DBI value found a significant correlation $(r=-0.0798, p<0.001)$. A diagnostic tests was performed between HIQ scores using a cut-off value $\geq 50$ with a $\mathrm{DBI}$ value $<0.6$ as a reference. It was obtained a sensitivity value of $15.3 \%$ and a specificity value of $100 \%$, with a diagnostic accuracy of $58.75 \%$.

Conclusion: There was a good correlation between HIQ and DBI in patients undergoing hemodialysis using upper arm native AVF. The use of HIQ as a screening tool in native upper arm AVF patients is less recommended, but its use for patients who are suggestive of stealing syndrome has good specificity results.

Keywords: digital brachial index, hand ischemic questionnaire, native arteriovenous fistule upper arm.

https://doi.org/10.36864/jinasvs.2020.1.006

*Correspondence: rsuhartono_md@yahoo.com

aM.D., Vascular and Endovascular Division, Department of Surgery, Cipto Mangunkusumo Hospital - Faculty of Medicine, Universitas Indonesia, Jakarta, Indonesia

bM.D., Department of Surgery, Ciawi Hospital, West Java, Indonesia

cM.D., M.T.I., Ph.D., Department of Community Medicine, Faculty of Medicine Universitas Indonesia, Jakarta, Indonesia

\section{INTRODUCTION}

End-stage renal disease (ESRD) is a terminal condition with increasing in prevalence. With the need for hemodialysis, making vascular access also causes other comorbidities in the patient, especially when the access experiencing complications. In the United States, $25 \%$ of patients with vascular access (arteriovenous fistula (AVF), AV graft, or central venous catheter) were treated for complications. ${ }^{1,2}$ Various complications that may
Copyright (C) 2020, The Indonesian Society for Vascular and Endovascular Surgery

JINASVS 2020;1(1):22-25 
hemodialysis. It had least re-intervention rate $(17 \%$, $\mathrm{p}<0.01)$, and steal syndrome rate $(0 \%, p=0.04)$ and the high level of functional patency of AVF after one year $(80.5 \%, p<0.001)$ compare to other types of AVF. ${ }^{4}$ Steal syndrome is a condition that is felt by patients as a sensation of cold, pain, cramps, and reduced sensibility. Although the incidence is small, severe stealing syndrome is estimated to be between $0.5-5 \% .{ }^{5}$ Risks for steal syndrome include the use of brachial arteries as inflow, diabetes, age $>60$ years, smokers, female sex, and history of repeated ipsilateral access. ${ }^{6}$

Although there was no gold-standard examination, there were various non-invasive predictors that can assess the degree of stealing, one of which is the digital brachial index (DBI) value. DBI of $<0.6$ is considered significant to assess the risk of stealing in patients. ${ }^{7,8}$ However, studies of DBI and steal syndrome are still quite limited. In this study, subjects with brachiocephalic AVF were assessed by hand ischemic questionnaire (HIQ) to determine its correlation with DBI.

\section{METHOD}

This study was a descriptive study using a cross-sectional design conducted in May - June 2019. Patients undergoing hemodialysis with upper arm native AVF access at Cipto Mangunkusumo Hospital, over 18 years old, and currently undergoing hemodialysis were included in the study criteria. Patients who had difficulty understanding language, cognitive impairments, and incomplete questionnaires were excluded from the study.

The selection of research subjects was done using the consecutive sampling method. DBI examination is performed on patients using the

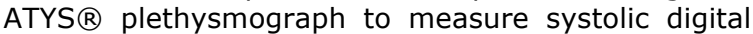
pressure. DBI measurement using a finger pressure monitor at the index finger. DBI values $<0.6$ were considered significant to assess the risk of stealing in patients.

\section{RESULTS}

This study involved 80 subjects undergoing hemodialysis with upper arm native AVF access at Cipto Mangunkusumo Hospital, which met the study criteria. The characteristics of the research subjects can be seen in table 1 .

Table 1. Characteristics of research subjects

\begin{tabular}{lcc}
\hline Type & $\mathrm{n}=80$ & $\%$ \\
\hline Gender & 37 & $46.2 \%$ \\
$\quad$ Men & 43 & $53.8 \%$ \\
$\quad$ Women & 53 & $(14-77)$ \\
Age, median (min-max) & & \\
Type of AVF & 73 & $91.2 \%$ \\
$\quad$ Brachiocephalic sinistra & 7 & $8.7 \%$ \\
$\quad$ Brachiocephalic dextra & & \\
Post Operation & 27 & $(6-118)$ \\
(month), median (min- & & \\
max) & & \\
Comorbidity & 20 & $25 \%$ \\
$\quad$ Diabetes mellitus & 70 & $87.5 \%$ \\
Hypertension & 2 & $2.5 \%$ \\
Polycystic kidney disease & $20 \%$ \\
$\quad$ Nephrotic syndrome & 8 & $10 \%$ \\
\hline
\end{tabular}

Research subjects were asked to complete a HIQ that assessed various symptoms of hand ischemia. From the results of normality test data, obtained abnormal distribution data $(p<0.05)$. Data are presented in table 2 . The results of the DBI normality test was a normal distribution $(p=0.171$, table 3).

Table 2. Characteristics of the HIQ

\begin{tabular}{ll}
\hline Variable & Median (min-max) \\
\hline Total of HIQ & $2,5(0-70)$ \\
Cold & $2,58(0-19)$ \\
Pain & $0(0-17)$ \\
Sensibility & $0(0-18)$ \\
Motoric & $0(0-18)$ \\
Cramps & $0(0-20)$ \\
\hline
\end{tabular}

Table 3. Characteristics of DBI.

\begin{tabular}{lll}
\hline Variable & Mean & CI 95\% \\
\hline $\begin{array}{l}\text { Digital Brachial } \\
\text { Index }\end{array}$ & $0.55 \pm 0,13$ & $0,52-0,58$ \\
\hline
\end{tabular}

From the correlation test obtained, DBI and HIQ had a strong correlation ( $p<0.001, r=-0.798$, figure 1).

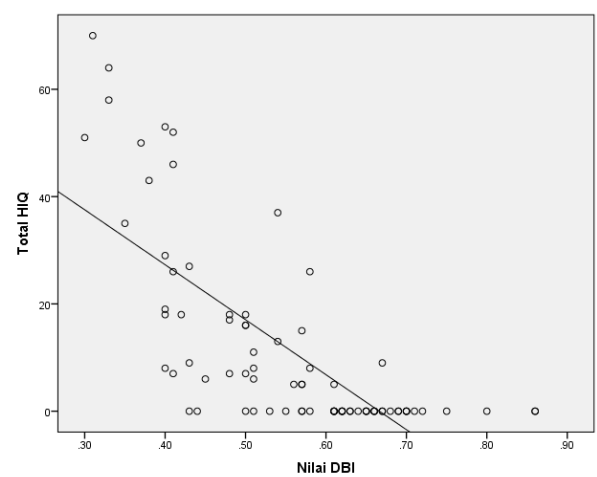

Figure 1. Random diagram of the correlation between HIQ and DBI values. Spearman correlation test analysis obtained a value of $p<0.001$ with a correlation strength of $r=-0.798(n=80)$.

The comparison between the results of the HIQ examination with DBI using a cut-off value of 0.6 was also analyzed. The comparison was assessed based on various HIQ categories based on the sum of the value, which includes normal, normal variation, and suggestive steal syndrome (table 4). Diagnostic tests were performed for HIQ, which were divided into $<50$ and $\geq 50$ with DBI values of 0.6 as a reference standard (tables 5 and 6 ).

\section{DISCUSSION}

This study aims to know the correlation between the hand ischemic questionnaire (HIQ) with the digital brachial index (DBI) of the arm with the upper arm AVF as access to hemodialysis in diagnosing steal syndrome. The HIQ score itself is a questionnaire that has a cut-off value of 50 to be suggestive of steal syndrome. The DBI is an examination to measure the comparison of digital arterial pressure with the brachial artery. DBI is considered superior to confirm the diagnosis of steal 
Table 4. Comparison of HIQ examination results with DBI above and below 0.6

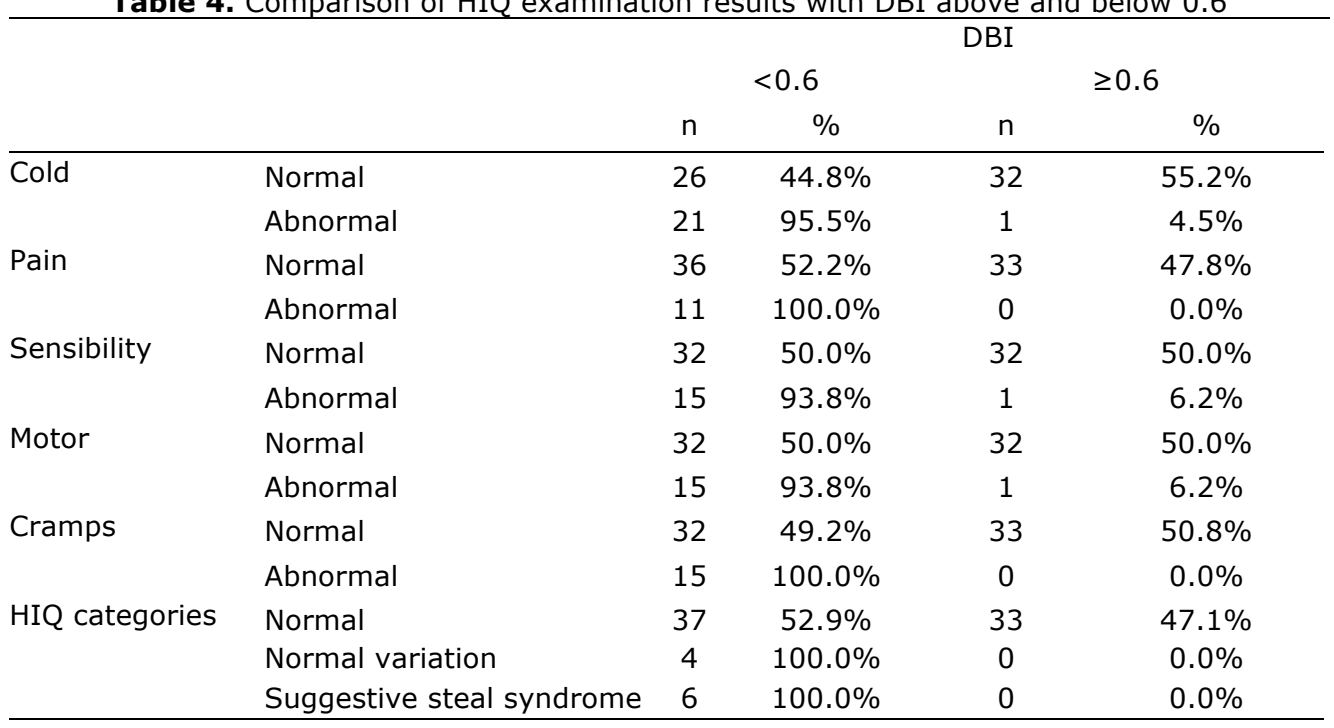

syndrome compared to other investigations. ${ }^{6}$ In this study DBI of $<0.6$ was used as a cut-off to determine which patients were suggestive of steal syndrome.

Table 5. Distribution of HIQ diagnostic tests $<50$ and $\geq 50$ against DBI values $<0.6$

\begin{tabular}{llll}
\hline & DBI $<0.6$ & DBI $\geq 0.6$ & Total \\
\hline HIQ $\geq 50$ & 6 & 0 & 6 \\
HIQ $<50$ & 33 & 41 & 74 \\
Total & 39 & 41 & 80
\end{tabular}

Based on the data obtained from the total score of the $\mathrm{HIQ}$, the minimum value was 0 , and the maximum value was 70 , with a median value of 3 . This finding showed that the total value of the HIQ score was much lower when compared with another study conducted by Van Hoek et al., ${ }^{9}$ who got a total HIQ score with a median of $258 \pm 30$. This was because there were different patient characteristics and examination methods. When compared with the previous study, they were using the HIQ questionnaire in hemodialysis patients who had clinically complained of ischemic symptoms in the hands of the fistula location. In contrast, in this study, the subjects of the study were all patients with ESRD who had undergone hemodialysis using an upper arm fistula with/without complaints of ischemic symptoms on the distal hand from the fistula location. The questionnaire filling method itself used the interview method because there were some difficulties in interpreting the terms intended in the questionnaire.

The results show that the HIQ questionnaire cannot be used as a tool for early detection of steal syndrome in a population of hemodialysis patients with native upper arm FAV without ischemic complaints at hand. However in patients who already have ischemic hand complaints, this questionnaire can be used because it has high specificity. This result also has been proven in several previous studies. ${ }^{6,8,10}$ This HIQ questionnaire can also be used to evaluate symptom improvement in patients with steal syndrome who have undergone FAV repair procedures. Research by Gerricken et al. found that the HIQ value of patients after undergoing the FAV procedure for steal syndrome was improving, from $155 \pm 33$ to $42 \pm 15$.

From the DBI results, the smallest number was 0.36 , and the biggest was 0.86 , with a mean of 0.57 , where the number was lower than 0.6 , which was used as a cut-off in this study. However, it did not rule out the patient who did not have experience of the early symptoms of steal syndrome. This result was seen from 37 patients $(52.9 \%)$ of this study with a normal HIQ score $(\leq 35)$ but had a low DBI result $<0.6$. If the cut-off is reduced to $<0.4$, then the number of patients in the normal HIQ category with abnormal DBI decreases dramatically to only seven patients. The procedure for assessing DBI also needs attention because photoplethysmography is very sensitive to light, temperature, and movement. The diagnostic test results between HIQ scores using a cut-off value $\geq 50$ with a DBI value $<0.6$ as reference standard resulted in a sensitivity value of $15.3 \%$ and a specificity value of $100 \%$, with a diagnostic accuracy of $58.75 \%$. It was known that HIQ was not recommended as a screening tool for hemodialysis patients in general.

This study has several weaknesses, including the presence of bias in the selection of subjects and the method of the examination carried out. Further research is recommended to be performed only on native fistula arms patients undergoing hemodialysis without symptoms of steal syndrome so that the bias could be minimalized. The patient also should fill the available questionnaire directly without being influenced by the explanation from the researcher.

\section{CONCLUSION}

Table 6. HIQ diagnostic test results using DBI as reference standard.

\begin{tabular}{llllll}
\hline & Sensitivity & Specificity & PPV & NPV & Accuracy \\
\hline HIQ to DBI & 15,38 & 100 & 100 & 55,4 & 58,7 \\
& $(7,2-29,7 \%)$ & $(91,4-100 \%)$ & $(61-100 \%)$ & $(44,1-66,2 \%)$ & $(47,8-68,9 \%)$ \\
\hline
\end{tabular}

PPV: positive predictive value, NPV: negative predictive value 
There was a good correlation between Hand Ischemia Questionnaire (HIQ) scores and Digital Brachial Index (DBI) in patients undergoing Hemodialysis using Upper Arm Native Fistula. The use of HIQ as a screening tool in native upper arm AVF patients is less recommended, but its use for patients who are suggestive of stealing syndrome has good specificity results.

\section{ACKNOWLEDGMENTS}

The author states the original work, and there is no conflict of interest in doing this research.

\section{ORCID ID OF AUTHORS}

Raden Suhartono

https://orcid.org/0000-0002-9142-3062

Muhamad Relly Sofiar

https://orcid.org/0000-0002-4846-6454

Aria Kekalih

https://orcid.org/0000-0001-7811-097X

\section{REFERENCES}

1. Simon $E$, Long $B$, Johnston $K$, Summers $S$. A case of brachiocephalic fistula steal and the emergency physician's approach to hemodialysis arteriovenous fistula complications. J Emerg Med. 2017 Jul;53(1):66-72.

2. Long B, Koyfman A, Lee CM. Emergency medicine evaluation and management of the end stage renal disease patient. Am J Emerg Med. 2017 Dec;35(12):1946-1955.

3. Daugirdas JT, Depner TA, Inrig J, Mehrotra R, Rocco MV, Suri RS, Weiner DE, et al. KDOQI clinical practice guideline for hemodialysis adequacy: 2015 update. Am J Kidney Dis. 2015;66(5):884-930

4. Chisci E, Harris LM, Menici F, Frosini P, Romano E, Troisi N, et al. Outcomes of three types of native arteriovenous fistula in a single center. J Vasc Access. 2017 Sep 11;18(5):379-383

5. Van Hoek F, Scheltinga MR, Kouwenberg I, Moret KE, Beerenhout CH, Tordoir JH. Steal in hemodialysis patients depends on type of vascular access. Eur J Vasc Endovasc Surg. 2006 Dec;32(6):710-7

6. Beathard GA, Spergel LM. Hand ischemia associated with dialysis vascular access: an individualized access flow-based approach to therapy. Semin Dial. 2013 May-Jun;26(3):287-314

7. Tynan-Cuisinier GS, Berman SS. Strategies for predicting and treating access induced ischemic steal syndrome. Eur J Vasc Endovasc Surg. 2006 Sep;32(3):309-15

8. Valentine RJ, Bouch CW, Scott DJ, Li S, Jackson MR, Modrall JG, Clagett GP. Do preoperative finger pressures predict early arterial steal in hemodialysis access patients? A prospective analysis. J Vasc Surg. 2002 Aug;36(2):351-6.

9. Vaes RH, Tordoir J, Scheltinga M. Blood flow dynamics in patients with hemodialysis access-induced hand ischemia. Journal of Vascular Surgery [Internet]. 2013 [cited 4 March 2019];58(2):446-451.e1.

10. Malik J, Slavikova M, Maskova J. Dialysis access-associated steal syndrome: The role of ultrasonography. Journal of Nephrology. 2003;16(6):903-7. 